\title{
Ethical Considerations in Face Transplantation: Ethical Issues Related to Inclusion Criteria for Face Transplant Candidates
}

\author{
Maria Siemionow
}

Received: 15 March 2011 / Accepted: 17 March 2011 / Published online: 27 March 2011

(C) L. Hirszfeld Institute of Immunology and Experimental Therapy, Wroclaw, Poland 2011

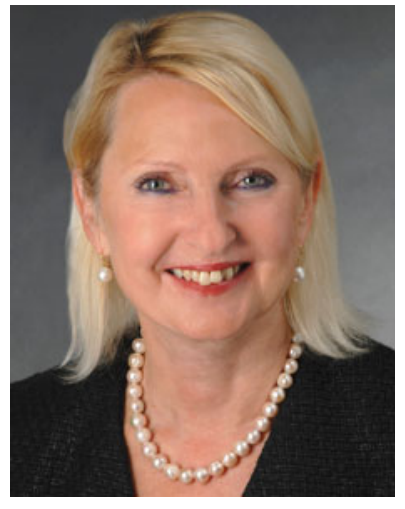

On November 15, 2004, Cleveland Clinic Foundation's Institutional Review Board (IRB) granted me the world's first approval to perform face transplantation in humans. This approval was granted based on over 20 years of work in the field of composite tissue allotransplantation, both in the experimental laboratory as well as in the anatomy laboratory, on cadaver models. The process of approval took over 1 year and a number of meetings in front of the IRB committee, which consisted of about 20 members of different specialties including medicine, transplantation, infectious disease, and surgery; however, also experts in different fields-lawyers, social workers, as well as lay representatives, all having the right to ask questions about the validity of such a new procedure.

When it comes to surgical breakthroughs, there is always a lot of controversy around the topic. It is quite

M. Siemionow $(\varangle)$

Department of Plastic Surgery, Cleveland Clinic,

9500 Euclid Avenue/Desk A-60, Cleveland, OH 44195, USA

e-mail: siemiom@ccf.org understandable that the announcement of Cleveland Clinic having received approval for facial transplantation in humans, in 2004, raised considerable interest from media all over the world, and from this perspective, I must say that the international and national media interest was almost larger than when we actually performed the first US face transplantation in December 2008. Interestingly, there was a lot of support from the media perspective, since it was obvious that our IRB approval was based on years of work in the field of composite tissue allograft transplantation. Also, the medical society supported the idea that, for patients who had lost their faces and were severely disfigured, introduction of new reconstructive options which went beyond what was currently available, was something to be accepted with interest and excitement.

The major opponents, however, of the idea of facial transplantation, were experts in ethics. One of them, Dr. Art Caplan, questioned the value of face transplantation and had numerous public interviews where he criticized the idea, as well as having warned society that now patients would be unwilling to donate organs and would be tearing up their drivers' licenses since, in the United States, organ donation is voluntary and a statement indicating agreement to be an organ donor is displayed on drivers' licenses. Interestingly, from the surgeons' perspective, this type of approach could be ethically questionable, specifically when it comes to statements made ex cathedra, without really dealing with disfigured patients and seeing their disability, daily suffering, and inability to maintain physiological functions, as well as normal social lives.

This introduces the ethical questions which we had raised during the work on our IRB approval protocol, as well as on the consent form for face transplant candidates. As for any medical procedure, when it comes to a decision about elective and novel surgery, there is always the 
question about exclusion and inclusion criteria under which particular patients will fit. This issue alone raises an ethical question of whether we are considering patients who will be included and given the chance to participate in the study and those who will be excluded based on, for example, their age, the fact that they smoke, abuse alcohol, or have a history of cancer. Interestingly, in published articles on the ethics of face transplantation, the issue of patient's inclusion criteria has only been briefly addressed (Agich and Siemionow 2004, 2005; Johnson and Corsten 2009; Kalliainen 2010; Paradis et al. 2010; Siemionow et al. 2009; Siemionow and Gordon 2010; Wiggins et al. 2004). Thus, I have chosen to share some of the ethical concerns which were raised when working on patient inclusion criteria for our IRB protocol. Based on these concerns, and understanding the limitations of any research study design, we have outlined, in our protocol, as well as in the consent form, patient inclusion criteria and examples of ten most important criteria are presented and discussed below.

1. The subject must be willing to sign the informed consent and agree to all follow up procedures including a realistic understanding of the impact of face allotransplantation on their lives.

The expectation that the patient has to have a realistic understanding of face transplant impact on their lifestyle was unknown at the time of our protocol approval, since there had not yet been any face transplantation performed, worldwide. Thus, from an ethical point of view it would be difficult to justify that the patient has a true understanding of the influence of this novel procedure.

2. Subjects will be evaluated by a plastic surgeon to assess implications for facial allotransplantation, based on the severity and complexity of the facial deformity.

When it comes to the plastic surgeon's assessment for facial transplantation, based on the complexity of facial deformity, this could, in some circumstances, raise an ethical question of whether someone, the physician or the team of experts, would approve patients who otherwise would not require facial transplantation, based on deformity, just to be the 'first' or to get notoriety in the field.

3. Subjects aged $18-60$ will be considered.

The age criteria alone could raise an ethical question of whether someone who is 17 or someone who is 61 , would not be a good candidate for face transplantation, thus indicating that the limitation of age may not always be equal for different types of patients.

4. The subject must be willing to undergo psychiatric and social pre-transplant evaluation.

This statement seems to be a logical request before a new experimental procedure, however, many patients who are on different types of medication or under the influence of substances, may be unwilling to go through the psychiatric evaluation and thus, will not be considered as a face transplant candidate.

5. The subject must be willing to undergo major facial surgery.

This seems to be a straightforward statement, however, for patients who are not educated in the medical field or do not have a higher level of general education with an understanding of what major surgery means, this may not be as clear as for those who have already undergone different types of surgeries or who read or search the topic via computer access and are familiar with the medical field.

6. The subject must be a candidate for general anesthesia. This, again, is a pretty straightforward question asked before a surgical procedure, but understanding that general anesthesia incurs potential significant risks including death, should be presented and understood by the patient, as well as the fact that it is difficult to predict how long the surgery will take, potentially exposing patients to the side effects of anesthesia of an unpredictable duration.

The six examples outlined above demonstrate the fact that some requirements and statements which seem to be straightforward in medical practice and apply to many surgical procedures, may still raise potential ethical concerns and questions when evaluating inclusion criteria for face transplantation, which is considered an experimental procedure.

The next set of inclusion criteria presented below applies to the field of organ transplantation, however, since the face is considered as a non-vital organ, the ethical concerns may be well justified and examples of such concerns are presented below.

7. The subject must be willing and able to receive potent drugs that induce immunosuppression and follow the infection prophylaxis protocol.

This may be a very difficult topic, from an ethical point of view, to present to potential face transplant patients since an understanding that some of the medications can generally shorten their life expectancy or deteriorate the function of many organs, such as the kidney or liver, may be difficult for a lay person to comprehend. Specifically, this applies to face transplant candidates, who are generally healthy patients in contrast to solid organ transplant patients, who are usually very sick and understand the risks and trade-off of lifelong immunosuppression in return for transplanted organ function. The same is true to understand the need for taking medications 
against transplant-related bacterial, viral, and fungal infections and the fact that all transplant patients are exposed to the side effects of these medications and as such, may be more prone to development of severe or even fulminant infections which are not common in patients who are not under immunosuppressive protocols. Thus, the ethical question arises if the presented risks are balanced by the benefits of face transplant surgery and the functional outcome.

8. The subject must be willing to receive standard vaccinations such as influenza, pneumococcus, and hepatitis B.

In the normal population, a person has the choice to decide whether or not to take the influenza, pneumococcal, or hepatitis B vaccinations. However, in the case of transplant patient candidates, it is important that they will receive these vaccinations before the surgery since they are more prone to developing severe viral infections, once they are submitted to a lifelong immunosuppression protocol, after transplantation.

Again, the need to comply with very specific protocols prior to transplantation surgery may sometimes raise the ethical question of whether the patient really understands the true risks, as well as true benefits of these vaccinations, and the fact that he may have no freedom to decide against vaccination as long as he wants to be considered for face transplantation.

9. The subject must be a non-smoker.

It is well known that wound healing is delayed in patients who smoke. This is also true for many procedures known as free flaps or free tissue transfers, to which the face transplant applies, since the side effects of smoking may cause a delay in wound healing or vessel constriction, compromising blood circulation to the graft and leading to tissue or graft failure. When planning for face transplantation, this is an important question to ask since if a patient who smokes understands the risks and is unable to quit smoking before transplantation, this raises two significant concerns: first, that the patient may be non-compliant when it comes to other commitments to be made after face transplantation, such as e.g., taking lifelong immunosuppression and second; it means that the patient does not understand the complexity and importance of collaboration with the team of physicians who are willing to guide the patient with what is best for him in order to reduce the risks of failure without taking away the patient's autonomy to make decisions.
On the other hand, a separate ethical question can be raised as to whether someone who cannot quit smoking, but is severely disfigured, should be denied face transplantation, considering the increased risk of transplant failure.

10. The subject must be free of malignant tumors for 5 or more years, with the exception of benign skin cancer. This is an important restricting criterion since someone who has active cancer will be at risk to tolerate the lifelong immunosuppression required after face transplantation. However, the ethical question arises as to whether someone who is severely disfigured and suffering, but also has cancer, has a right to decide whether he would be willing to accept the potential side effects of immunosuppression, including progression of cancer and shortened life expectancy, in exchange for a few years of better quality of life and function expected after face transplantation which would be performed to alleviate their suffering from the disfigurement.

The above examples of face transplant candidates' inclusion criteria, selected from the face transplant protocol and consent form, indicate that even with the best intention to comply with Hippocrates oath-Do not harm-the ethical debate will continue since there are really no fully justified inclusion or exclusion criteria from an ethical point of view.

\section{References}

Agich GJ, Siemionow M (2004) Facing the ethical questions in facial transplantation. Am J Bioeth 4:25-72; discussion W23-31

Agich GJ, Siemionow M (2005) Until they have faces: the ethics of facial allograft transplantation. J Med Ethics 31:707-709

Johnson SE, Corsten MJ (2009) Facial transplantation in a new era: what are the ethical implications? Curr Opin Otolaryngol Head Neck Surg 17:274-278

Kalliainen LK (2010) Supporting facial transplantation with the pillars of bioethics. J Reconstr Microsurg 26:547-555

Paradis C, Siemionow M, Papay F et al (2010) Ethical considerations in the first American face transplant. Plast Reconstr Surg 126:896-901

Siemionow MZ, Gordon CR (2010) Institutional review board-based recommendations for medical institutions pursuing protocol approval for facial transplantation. Plast Reconstr Surg 126:1232-1239

Siemionow M, Papay F, Alam D et al (2009) Near-total human face transplantation for a severely disfigured patient in the USA. Lancet 374:203-209

Wiggins OP, Barker JH, Martinez S et al (2004) On the ethics of facial transplantation research. Am J Bioeth 4:1-12 